\title{
MULTI-FEATURE SPARSE CONSTRAIN MODEL FOR CROP DISEASE RECOGNITION
}

\author{
WU, Y. R. ${ }^{1}-\mathrm{LI}, \mathrm{J} . \mathrm{H}^{2 *}$ \\ ${ }^{\text {I}}$ Zhongkai Science and Technology Development Company, Zhongkai University of Agriculture \\ and Engineering, Guangzhou 510225, China \\ ${ }^{2}$ The Laboratory of Language Engineering and Computing, Guangdong University of Foreign \\ Studies, Guangzhou 510006, China \\ *Corresponding author \\ e-mail: lijianhonghappy@163.com \\ (Received $1^{\text {st }}$ Apr 2019; accepted $17^{\text {th }}$ May 2019)
}

\begin{abstract}
Disease recognition based on crop leaf image is considerably important and significant, which is a challenging project due to the complicated nature of disease leaf image itself and the unavoidable degradations sourced from external imaging environment. In the recognition process, these traditional approaches generally stack one or more different modalities of features extracted from disease leaf images to be discriminated or trained, but do not deeply explore the relationships between features. For conquering this problem, we proposed a new crop disease recognition approach based on multi-feature sparse constrain model, which includes three steps: lesion segmentation, feature extraction and disease recognition. During the phase of disease recognition, the characteristic of our proposed is that the introduced multi-task joint sparse representation model can promote these sparse representation vectors corresponding to the different modalities of features to share similar structures to some extent with the specific styles of features extracted from the same training set. The proposed approach not only improve the efficiency significantly but also make the decision more robust. We verify the proposed approach with images of diseased cucumber, and the experiments demonstrate that our proposed method is remarkable superior to the state of the art algorithms and the correct recognition rate of our approach is $88.05 \%$, which is higher $11.3 \%$ percentage than the benchmark of SVM method.
\end{abstract}

Keywords: crop disease identification, multi-task learning, joint sparse representation, accelerated proximal gradient, lesion segmentation, feature extraction

\section{Introduction}

Crop diseases have long been considered as one of the primary threats to reduce crop yield and quality. According to recent reports, the global plant yield has reduced by at least 10\% each year due to the crop diseases (Mutka and Bart, 2015). It can be seen that whether crop diseases can be discovered in time is significantly important for increasing the production and quality of agricultural products. However, the identification of crop diseases to date has remained on the basis of artificial judgment, which is inefficient and subjective. In China, due to the lack of professional agricultural technicians, farmers usually are forced to judge the type and evaluate its degree of diseases according to their past experience. The wrong diagnosis and the resulting pesticide abuse are extremely common as expected. Therefore, it is urgently to find a timely, accurate and low-cost approach to alleviate the present condition

Due to the huge driving force of the potential market and the rapid development of computer vision and machine learning technology, in recent years, crop disease identification has received widespread attention by researchers, and a large number of related algorithms have been proposed. Generally speaking, the common disease 
identification algorithms mainly include 4 steps (Iqbal et al., 2018; Golhani et al., 2018; Dhingra et al., 2019; Zhang et al., 2017b, 2018; Zhang and Wang, 2016), followed by disease image pre-processing, disease lesion segmentation, disease feature extraction, and disease species identification. In the pre-processing step, image enhancement operations, such as median filtering (Unay et al., 2011; Sun et al., 2018), histogram equalization (Khirade and Patil, 2015), etc., are usually adopted to avoid interference of notorious noise and uneven illumination in the sample image. Lesion segmentation refers to the localization process to find the lesion areas with pixel level in the leaf images. The commonly used algorithms include K-means algorithm (Zhang et al., 2018, 2017a; Ghosh and Dubey, 2013; Prajapati et al., 2016), OTSU algorithm (Zhang and Wang, 2016; Dey et al., 2016), and super pixel clustering (Zhang and Zhu, 2017). When the lesion area is separated, it is necessary to extract some modality features such as color, shape, texture or other attributes of the lesion. Whether the extracted features are appropriate or not directly determines the effectiveness of the disease identification algorithm. Common feature extraction methods include: Principle Component Analysis (PCA) (Zhang and Wu, 2017), wavelet analysis (Kleynen et al., 2005; Shaik et al., 2018), PSO (Muthukannan and Latha, 2015), gray level co-occurrence matrix (Iqbal et al., 2018) and so on. In general, the identification of a particular disease can use only one modality feature, however when distinguishing many kinds of diseases, mining the complementary information between the different modality features will obtain higher accuracy rate than just one. Disease identification is the final step of the approach which refers to taking advantage of the pattern recognition technology to classify the extracted features to achieve the purpose of identification. Commonly used disease classification algorithms include artificial neural networks (Bashish et al., 2011), support vector machine (Rumpf et al., 2010; Arivazhagan et al., 2013), K-nearest neighbor algorithms (Zhang et al., 2015), and sparse representation (Zhang and $\mathrm{Wu}, 2017$ ), so on. Meanwhile, we also found that with the rise of deep learning, this technology has also been applied in the field of crop diseases identification.

In theory, deep learning not only avoids lesion segmentation and feature extraction steps, thus brings great convenience into the designing algorithm process, but also improves the correct recognition rate significantly (Ma et al., 2018; Grinblat et al., 2016; Zhang et al., 2019; Sladojevic et al., 2016; Lu et al., 2017). However, it is coexisting that deep learning technology is extremely demanding on the quantity and quality of samples. Accurate identification of any kind of disease requires massive standard sample images, complex parameter debugging and abnormal time-consuming model training. In the process of training the deep learning model, even an additional hardware device - Graphic Process Unit (GPU) is necessary. Obviously, subjecting to these factors such as geography, season and cost, the harsh conditions almost impossible to achieve.

In response to the above-mentioned problems, we are committed to developing a new crop disease identification approach, which can accurately and swiftly determine the type of disease according to the appearance of leaf diseases image. At the same time, this approach is not limited to the quantity and quality of training samples to save the cost of collecting samples. We summarize the main contributions of our algorithm proposed in this paper as follows:

1. Based on the sparse theory, we propose a new crop disease identification algorithm. The algorithm uses the multi-feature sparse constrain to describe the proposed prior, constructs the cost function, and successfully optimizes the cost function with the algorithm of accelerated proximal gradient. By comparing the 
recognition efficiency of cucumber leaf disease, the recognition efficiency of the algorithm is obviously better than other state of the art algorithms.

2. Inspired by the idea of multi-task learning, we find a new prior, which points out that the different modalities of disease features can be represent with the corresponding features extracted from the training set. The structure of the sparse coefficient vectors should be similar. This proposed multi-feature sparse constrain model has a more realistic constraint effect, and the resulting sparse coefficient is more accurate, robust, and close to the actual situation.

3. Because there is no complicated training process and cumbersome parameter setting step in this paper, the principle of our algorithm is well comprehensible, thus its calculation process is simple and easy to operate. More unexpected is that the performance of our approach is still extraordinary excellent in the case of insufficient training samples. This characteristic can help us conquer the difficulty of the dependence on a large number of samples, which are susceptible to various factors for obtaining.

In the first section, we summarized the significance and status quo of automatic disease identification. Some related work to our algorithm will be introduced in the second part. The first will elaborate the proposed algorithm, including 4 steps: preprocessing, detection feature extraction and recognition. In the fourth part, we will give the comparison results of the proposed algorithm and other state of the art algorithms, and further analyze the performance of the algorithm. The last section is the conclusion and prospect of the paper, and it points out the unresolved problems in the fields of automatic disease identification and puts forward our working plan in the future.

\section{Related work}

At the beginning of this century, neuroscientists found in their research that for images entering in the human eye, the human visual system uses sparse representation to represent it with the help of as little as possible base feature (Rao et al., 2002). Inspired by this phenomenon, sparse representation has been widely used and deeply researched in machine learning and computer vision (Wright et al., 2009; Yang et al., 2010; Mei and Ling, 2011). The equation it needs to solve can be expressed as

$$
\underset{w}{\arg \min } \frac{1}{2}\|y-D \omega\|_{2}^{2}+\lambda\|\omega\|_{0}
$$

where y is a certain form of feature vector extracted from the input image, $D$ is an overcomplete dictionary composed of numerous base vectors, and the base vector in the dictionary is called "atom". In general, a dictionary can be constructed by extracting features of a corresponding form from a collection of sample images. $w$ is a sparse coefficient vector that needs to be solved and can sparsely represent the feature $y .\|w\|_{0}$ is $L_{0}$ norm represents the number of non-zero elements in the vector. However, Equation 1 is non-convex, and the solution is very difficult to find. It is usually necessary to relax the constraint and replace the $L_{0}$ norm in Equation 1 with the $L_{1}$ norm, thus to solve it with Lasso algorithm (Wright et al., 2009; Yang et al., 2010; Mei and Ling, 2011).

Image classification based on sparse representations shows great potential for crop disease identification (Zhang and $\mathrm{Wu}, 2017$ ). It does not require a large number of 
training samples, and can accurately identify the types of diseases for most "regular" diseased leaves. However, in the process of optimizing the cost function, the Lasso algorithm usually selects atoms in the disease "dictionary" by an approximately random way to fit the disease features to be tested. The robustness of the recognition algorithm makes the judgment unstable. At the same time, in the optimal computing process, it is possible to wrongly make some discriminative 'atom' participate in the computing thus lead to the recognition accuracy rate reducing. In addition, in the case of recognition disease by multi-modality of features, the image classification technique based on sparse representation just stacks features such as color, shape, texture or spatial distribution of the same sample indiscriminately into one vector in the process of feature extraction, without considering the correlation between different kinds of features. This is essentially equivalent to constrain the sparse coefficients of these different forms of features to be identical. Obviously, if the judgments with these different forms of features are separately, some ambiguous conclusions may be drawn; and these identical sparse vectors corresponding to that different modality features are separately contrary to the real situation, which will introduce new errors and affect the judgment.

In fact, if considering the disease recognition with some modality feature as a task, a large number of researches have shown that (Zhang et al., 2018, 2017a; Zhang and Wang, 2016; Unay et al., 2011; Sun et al., 2018; Khirade and Patil, 2015) when there are some commonalities among these being learned tasks, improving the recognition efficiency by mining the relative information is feasible (Ozawa et al., 2009; Argyriou et al., 2008; Bickel et al., 2008; Caruana, 1997), which is called multi-task learning or transfer learning (Caruana, 1997). In the field of machine learning and computer vision, when the related tasks are affected by some common factors, the ability of multi task learning or transfer learning to improve the generalization ability is significant by mining relationship among tasks especially in the case of insufficient training samples. The basic idea of this paper is that when we use sparse representation to describe a testing feature with $\mathrm{K}$ modalities, and the dictionary is constructed by the corresponding $\mathrm{K}$ modalities of features extracted from the same training set, thus for each modality, we can consider it as a task. The fact is that when the reconstruction error is smallest, the smallest number of 'atoms' from one class can linearly represent the multi-feature, which can be considered as the final classifying result.

\section{Materials and methods}

\section{Lesion segmentation and feature extraction}

Assuming that there are $J$ kinds of disease images in data set $X$, and $n_{j}$ samples in disease $j$, the total number of samples in disease set is $\sum_{j=1}^{J} n_{j}=N$. For each disease image, we extract $K$ different kinds of features such as color, shape, texture, etc. The training set can be expressed as

$$
X=\left[\begin{array}{cccc}
X_{1}^{1} & X_{2}^{1} & \cdots & X_{J}^{1} \\
X_{1}^{2} & X_{2}^{2} & \cdots & X_{J}^{2} \\
\vdots & \vdots & \cdots & \vdots \\
X_{1}^{K} & X_{2}^{K} & \cdots & X_{J}^{K}
\end{array}\right]_{M \times N}
$$


where $X_{j}^{k} \in R^{m_{k} \times n_{j}}$ represents the $k$-th class feature of the $j$-th type of disease image, and each column in $X_{j}^{k}$ is a feature vector with dimension $m_{k} . X^{k}=\left[X_{1}^{k}, X_{2}^{k} \cdots, X_{J}^{k}\right]$ is a matrix of $m_{k} \times N$ representing the $k$-th class feature of the disease set. $X_{j}=\left[\left(X_{j}^{1}\right)^{T},\left(X_{j}^{2}\right)^{T}, \cdots,\left(X_{j}^{K}\right)^{T},\right]^{T}$ is a matrix of $M \times n_{j}$ representing the disease features of $n_{j}$ samples in the $j$-th disease, and the dimension of each disease feature vector is $\sum_{k=1}^{K} m_{k}=M$. For a diseased leaf image to be tested, we extract its $K$-modalities features $y^{k} \in R^{m_{k}},(k=1,2, \ldots, K)$, and the feature vector of the diseased leaf image to be tested can be expressed as $Y=\left[\left(y^{1}\right)^{T},\left(y^{2}\right)^{T}, \cdots,\left(y^{K}\right)^{T}\right]^{T}, Y \in R^{M}$. For the feature vector $Y$, we hope that each $y^{k}$ can be sparsely represented by the corresponding feature $X^{k}$ in the training set, and the obtained $K$ sets of coefficients should have similar spare structure. The mathematical representation of this idea can be described in Equation 3.

$$
\arg \min _{w} \frac{1}{2} \sum_{k=1}^{K}\left\|y^{k} \sum_{j=1}^{J} X_{j}^{k} W_{j}^{k}\right\|_{2}^{2}+\lambda\left\|\left(\left\|W_{1}\right\|_{F},\left\|W_{2}\right\|_{F}, \cdots,\left\|W_{J}\right\|_{F}\right)\right\|_{0}
$$

where $W_{j}^{k} \in R^{n_{j}}$ represents the coefficient vector corresponding to the feature in the $k$-th form of the $j$-th disease image set, and the matrix $W_{j}=\left[W_{j}^{1}, W_{j}^{2}, \cdots, W_{j}^{k}\right], W_{j} \in R^{n_{j} \times k}$, vector $W^{k}=\left[\left(W_{1}^{k}\right)^{T},\left(W_{2}^{k}\right)^{T}, \cdots,\left(W_{J}^{k}\right)^{T}\right]^{T}, W^{k} \in R^{n},\|W\|_{1,2}=\sum_{i=1}^{n}\left\|R_{i}(W)\right\|_{2}$, and $R_{i}$ are responsible for extracting the $i$-th row of the coefficient matrix $W$. The concrete expression of the coefficient matrix is

$$
W=\left[\begin{array}{cccc}
W_{1}^{1} & W_{1}^{2} & \cdots & W_{1}^{K} \\
W_{2}^{1} & W_{2}^{2} & \cdots & W_{2}^{K} \\
\vdots & \vdots & \cdots & \vdots \\
W_{I}^{1} & W_{J}^{2} & \cdots & W_{J}^{K}
\end{array}\right]_{n \times k}
$$

where $\left\|W_{j}\right\|_{F}$ refers to the Frobenius norm of the matrix $W_{j}$, and $\|x\|_{0}$ refers to the $L_{0}$ norm of the vector $\mathrm{x}$, that is the number of non-zero elements in the vector $\mathrm{x}$. However, the $L_{0}$ norm in Equation 3 makes the cost function non-convex, and to find the solution is very difficult. In order to solve the problem, we relax the cost function to Equation 5

$$
\arg \min _{w} \frac{1}{2} \sum_{k=1}^{K}\left\|y^{k}-X^{k} W^{k}\right\|_{2}^{2}+\lambda \sum_{j=1}^{J}\left\|W_{j}\right\|_{F}
$$

We call Equation 5 a multi-feature sparse constrain model. Its solution is simple to solve. We use the accelerated proximal gradient method to solve the problem. For detailed solution steps, please refer to Algorithm 2 in this paper.

\section{Multi-feature sparse constrain}

The accurate detection of the lesion area and extracting appropriate features play an important role in the disease identification process. The $K$-means clustering algorithm is one of the classic algorithms for image segmentation. It divides all the pixels of the input image into $K$ classes according to the feature of each pixel. Since the content of the disease image is relatively fixed, it can generally be regarded as consisting of a healthy area, a diseased area, and a transitional area between the two. Therefore, the 
determination of $K$ and the selection of cluster centers are relatively easy. In this paper, we use the $K$-means clustering algorithm to segment disease images. The RGB value of each pixel is taken as its feature vector. The core idea of the k-means algorithm is to divide $n$ RGB vectors into $k$ clusters, so that the sum of the squares of the distance between the RGB vector in each cluster and the cluster center is the smallest. The algorithm flow is as shown in Algorithm 1.

\section{Algorithm 1. K-means algorithm segmentation}

1. Select $K$ Pixels as the initial cluster centers randomly;

2. Calculate the distance between the remaining samples and each cluster center, classify the sample into this class with the nearest distance;

3. Re-compute the average value of each class and update the clustering center of each class with the criterion function

$$
\mathrm{E}=\sum_{i=1}^{C} \sum_{x \in C_{i}}\left\|x-x_{C_{i}}\right\|^{2}
$$

4. When the criterion function no longer converges, the clustering result is output, otherwise it is repeated Step2 Step4.

The $K$-means algorithm divides all pixels in the disease image into $K$ categories. Next, you need to find the class of the pixel in the disease area accurately in this $k$-type pixel. In fact, because the characteristics of different diseases on the leaves are different, it is very difficult to find out the common characteristics of various diseases and directly locate the class of the diseased pixels. However, the characteristics of the healthy area of the leaf are easy to find and stable from the perspective of color and texture. To this end, we find the pixels of the healthy area from the $K$-type of pixels, and the remaining pixels are considered to be the pixels of the diseased area. This method will judge some of the transitional pixels between the healthy area and the diseased area as the disease content, so that some initial characteristics of the disease can be reflected from the information provided by the disease area, on the one hand, the early symptoms can be reflected in the extracted features, and on the other hand, the efficiency of recognition of the overall disease is also improved. Figure 1 shows the segmentation results of four disease images. It can be seen from the results given that the pixels of most disease areas can be correctly found by the $K$-means algorithm. After locating the disease region, we will extract feature for the disease areas. 4- Unicom disease region is first determined region area, and further select intermediate 4- Unicom disease region to extract features. In this paper, we consider the three aspects of color, shape and texture to extract the feature of the diseased area. In order to avoid the affection of scale and direction, we try to select some features that will not disturbed by the scaling and rotation factors. We have selected a total of 28 features, including 4 color features, 6 shape features, and 18 texture features. The specific feature name and the corresponding feature can be found in the Appendix. After normalizing the different types of feature vectors, they connected each other to construct the feature vector we need to use.

\section{Disease identification}

The process of disease classification is relatively simple. For each type of disease $j$, Equation 6 is used to calculate the reconstruction error between the testing feature and its fitting result, and select $j^{*}$ corresponding to the smallest error as the decision. 


$$
j^{*}=\arg \min _{j} \sum_{k=1}^{K} \theta^{k}\left\|y^{k}-X_{j}^{k} W_{j}^{k}\right\|_{2}^{2}, \mathrm{j}=1,2, \cdots, \mathrm{J}
$$

where $\left\{\theta^{k}\right\}_{k=1}^{K}$ are the weights of the different feature forms in the recognition process. According to the definition, we will elaborate weight parameter $\left\{\theta^{k}\right\}_{k=1}^{K}$ in the experimental part. Further, the disease category $j^{*}$ which minimizes the error of the reconstructed test disease feature is selected as the final recognition result. The pseudo code of the process is shown in Algorithm 2.

Algorithm 2. Multi-task joint sparse representation

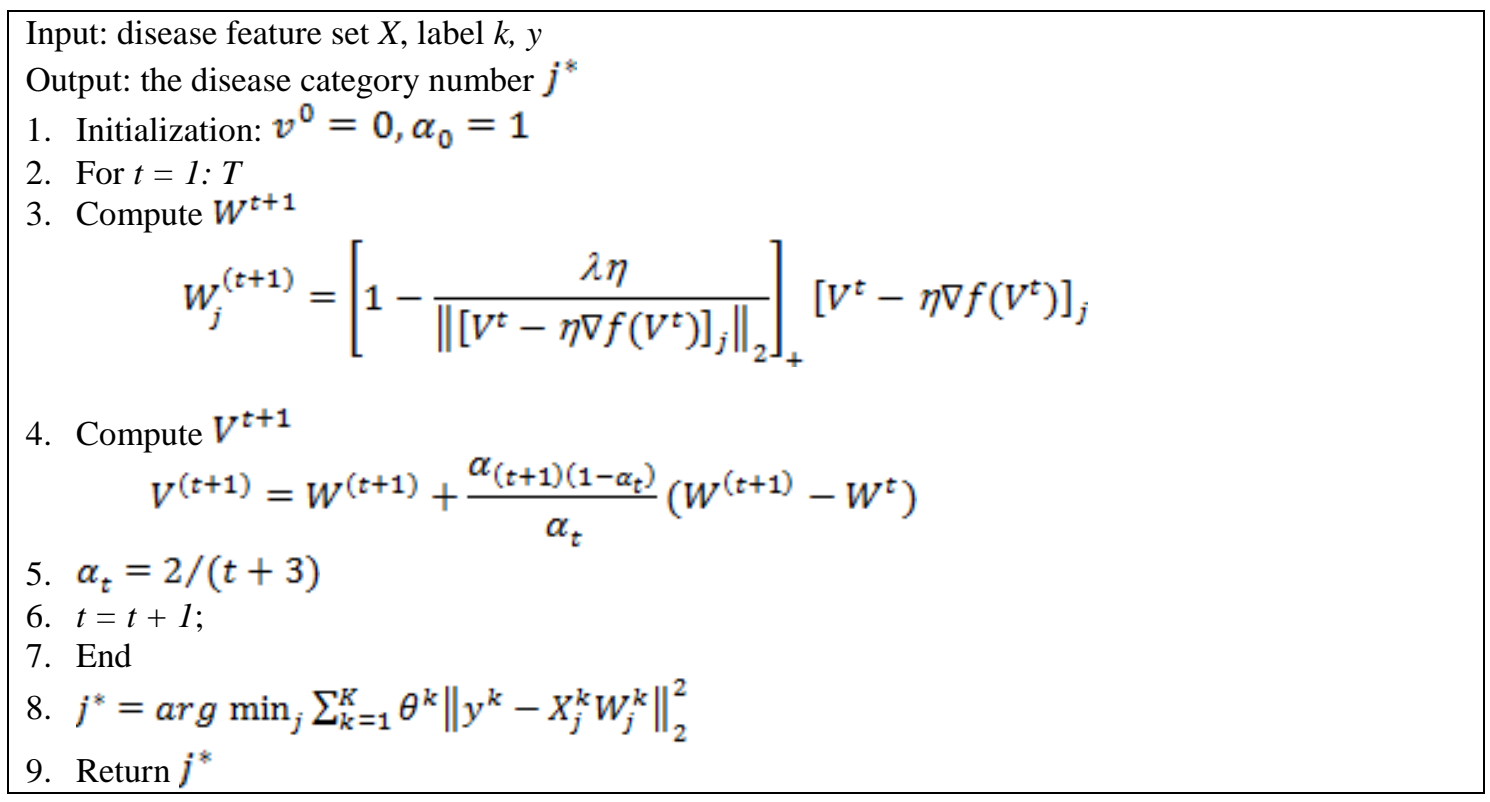

\section{Experiment and analysis}

In order to verify the effectiveness of the proposed algorithm, we conducted extensive experiments on cucumber leaf diseases image and do comparisons with state of the art results published in recent years. These state of the art algorithms include SR (Zhang and Wu, 2017), SVM (Rumpf et al., 2010), ANN (Bashish et al., 2011), TF (Arivazhagan et al., 2013), and KNN (Zhang et al., 2015). All experiments were performed on a computer with $1.8 \mathrm{GHz}$ CPU and $2 \mathrm{~GB}$ cache and a 64bit Windows $2 \mathrm{~K}$ operating system was installed in it. All the algorithms including ours proposed in this paper were coded and implemented on MATLAB2015b platform. The results of the participating comparison algorithm are basically consistent with the results given in the experimental part of the corresponding paper.

\section{Parameter settings}

The cucumber leaf disease image used in this paper are collected in the farm of Zhongkai University of Agricultural and Engineering. All the disease leaves are obtained by agronomy students under the guidance of professional teachers through digital cameras or smart phones, and the corresponding disease types are labeled by agriculture experts. In the process of obtaining examples, in order to avoid the interference of uneven illumination, atmospheric turbulence and other factors, our 
image acquisition process is completed indoors, and set the same shooting environment for each image. All captured images are set to $800 \times 800$ pixels by cutting or zooming, etc. All images are three-channel color images and saved as "jpg" format. A partial images selected form the data set is shown in Figure 2. The data set contained 1,120 images with seven common diseases, i.e., downy mildew, bacterial angular, Corynespora cassiicola, scab, gray mold, anthracnose, powdery mildew. Each disease contained 160 samples and for each disease, we selected 100 samples randomly for training in our model, and the remaining 60 examples were used for testing. Considering that there may still exist noise or uneven illumination introduced during the shooting process, we perform smoothing and histogram equalization before starting the diseased region segmentation step. Next, extract the features of each disease image according to the explanation in Section 3.2, and add labels for each example to construct a training data set. Further, the sparse coefficient $\mathrm{W}$ is obtained by the Equation 5, and the type of the disease is judged by the Equation 6. The feature weight parameters in Equation 6 are set to: color weight $\theta^{1}=0.5$, shape weight $\theta^{2}=0.3$, texture weight $\theta^{3}=0.2$, and the settings will be explained in next sub-section.
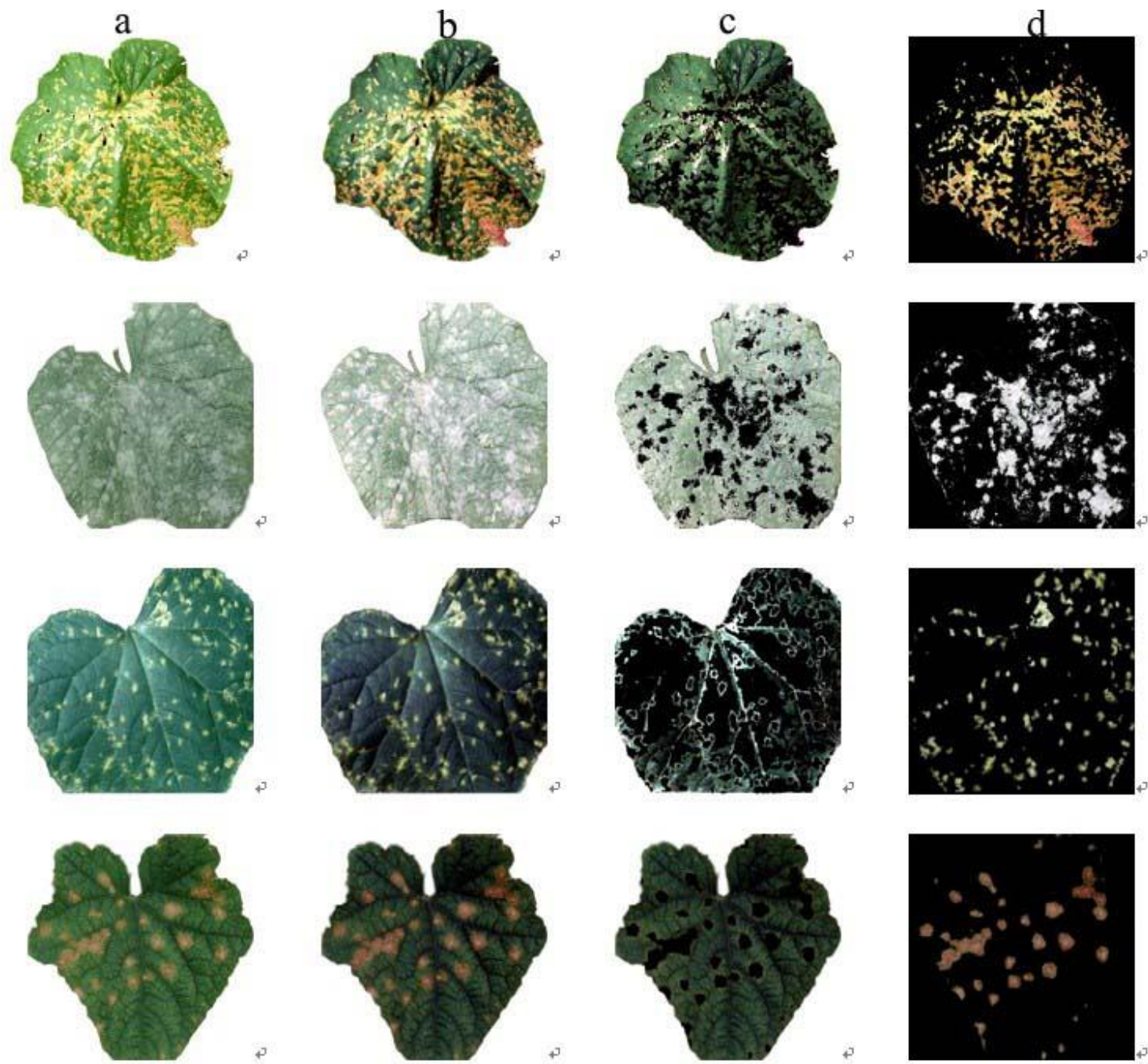

Figure 1. The results of healthy region and legion region with K-means segmentation (the disease names from the top line to the final line are bacterial angular, powdery mildew,

Corynespora cassiicola and anthracnose separately). (a) Disease leaf images; (b) Enhancement leaf image; (c) Healthy region; (d) Legion region algorithm 

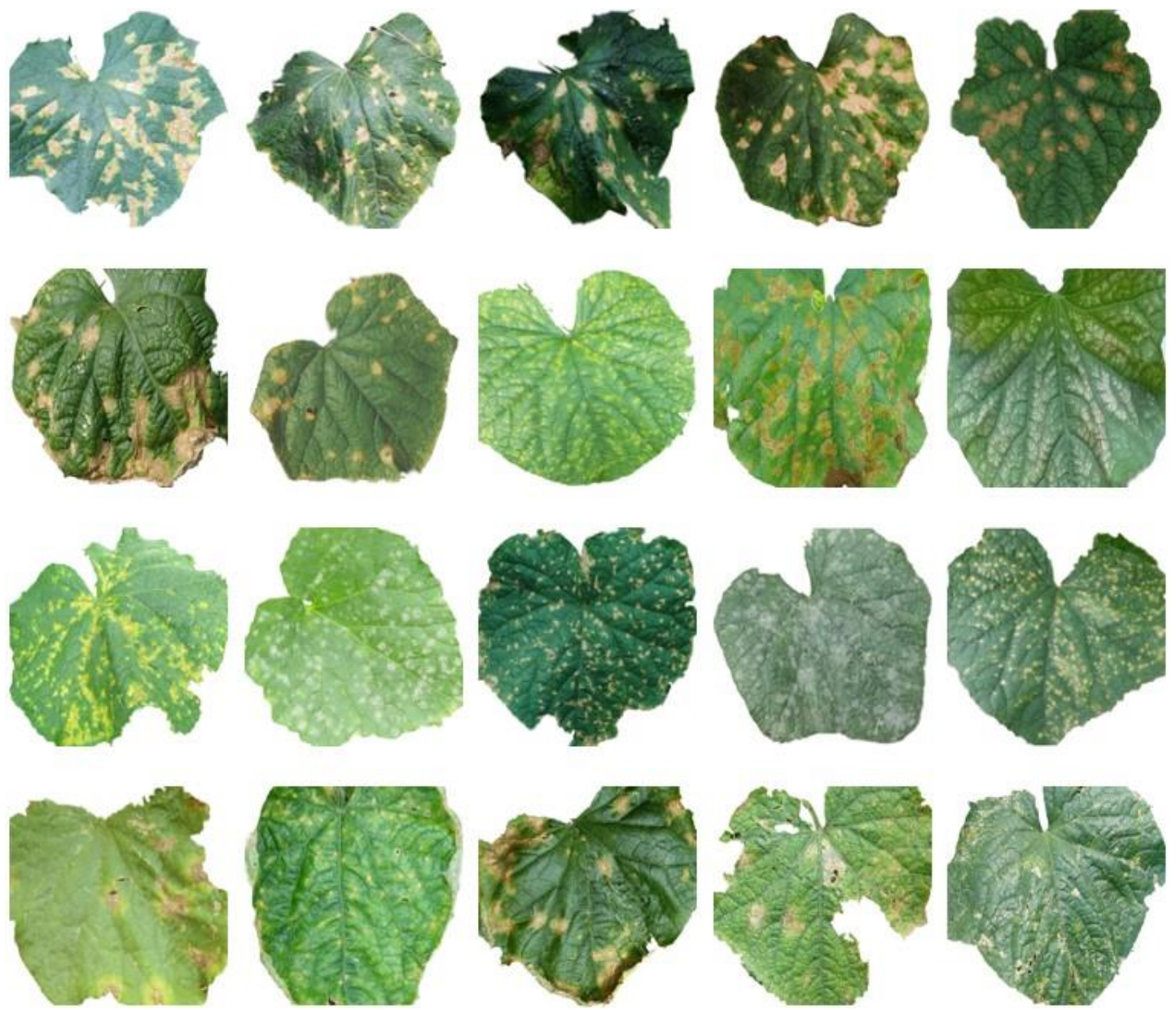

Figure 2. Partial cucumber disease leaf examples

According to Figure 3, we can conclude that the accuracy of our method is obviously better than the sparse representation with a single feature and better than other state of the art algorithms without considering the relationship between features such as the algorithm of (Zhang and $\mathrm{Wu}, 2017)$.

\section{Experimental results}

After obtaining the training and testing data, we randomly selected a certain number of samples in each type of disease image as "atoms" to construct a dictionary to identify the disease of testing example. The results are shown in Figure 3. In the case of considering only one feature, the method degenerates into a sparse representation recognition algorithm. Since only one feature form is considered, its recognition efficiency is low. The solution of sparse coefficient is similar to SR algorithm, but due to the single feature form, its recognition efficiency is lower than the SR algorithm. The SR algorithm uses sparse representation classification theory for disease identification, which just stacks color features, shape features and texture features indiscriminately. In the process of solving sparse coefficients, the SR algorithm randomly select as few as possible "atoms" to approximately fit the testing example which is unstable. However, our method comprehensively considers the sparse coefficient vectors of the 
corresponding different modalities features as similar as possible, which is a stronger constraint and makes the recognition results more accurate.

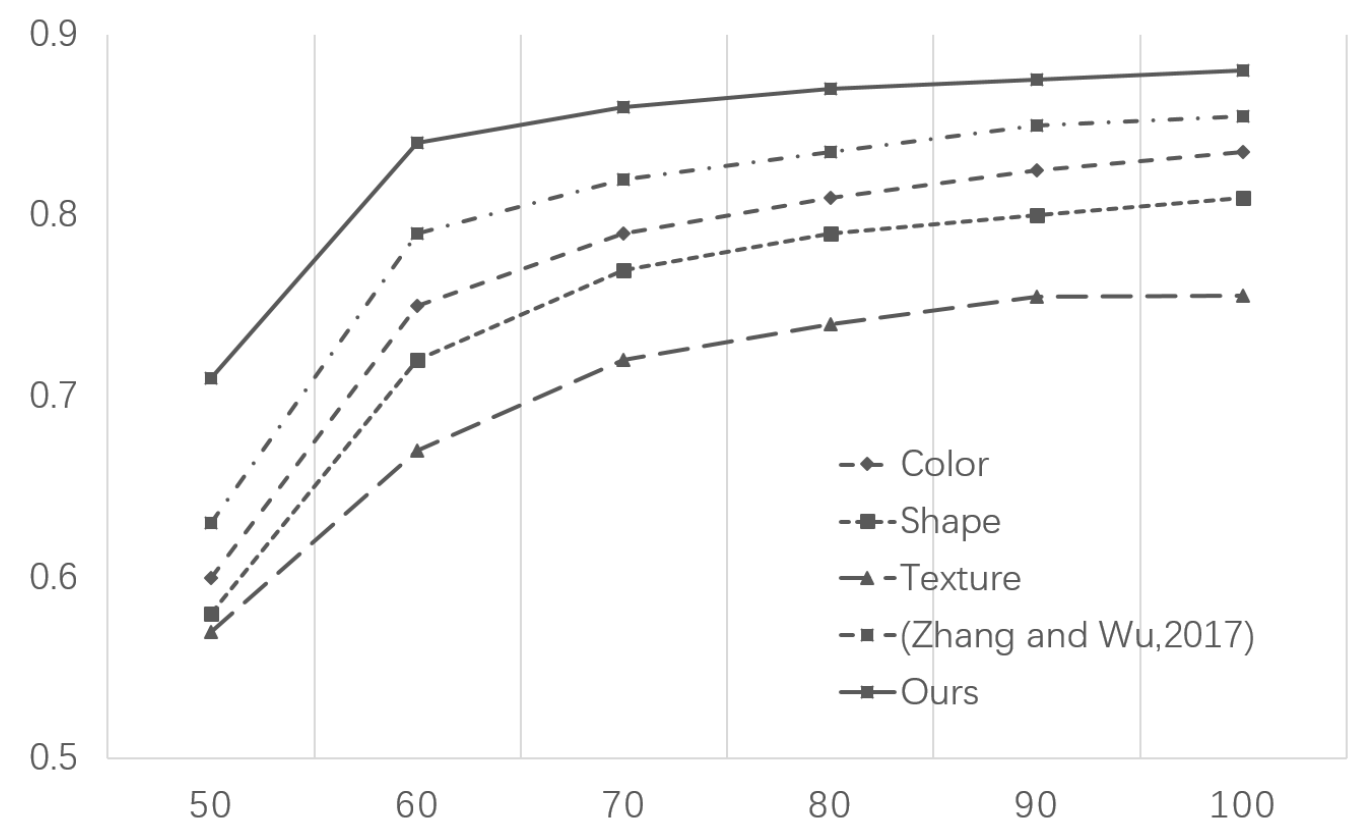

Figure 3. The accuracy curve of cucumber disease identification

At the same time, we can notice that our algorithm has increased the recognition efficiency as the number of training samples increases, and it is always higher than other methods.

Observing the experimental results given in Figure 3, we can see that when the three features of color, shape and texture are processed separately, the efficiency of each is not the same. In order to quantify the proportional relationship between them and determine the parameters $\left\{\theta^{k}\right\}$, we set the color and shape characteristics in the range of, and quantize, and the step size is set to 0.1. After the color feature weight and the shape feature weight are determined, the texture feature weight may not be displayed. However, for each set of weight values, a disease identification test was conducted to obtain an average accuracy rate, and the results are shown in Figure 4. It can be seen from Figure 4 that when $\theta^{1}=0.5, \theta^{2}=0.3, \theta^{3}=0.2$, the recognition rate reaches the maximum value, so other experiments in this paper are performed according to this setting.

Furthermore, according to the conclusion of Figure 4, we set the number of atoms in the dictionary to 700, (that means that 100 samples for each type of disease to participate in the training), and test the correct recognition rate and average consumption of the disease with the selected state of the art algorithms. The comparison results of the correct recognition rate of the disease are shown in Table 1, and the average time consumed by these algorithms is shown in Table 2. By observing the experimental data exhibited in Tables 1 and 2 , it is not difficult to find that our algorithm has the highest accuracy and the minimum average time to identify the results of the identification of these seven diseases. 


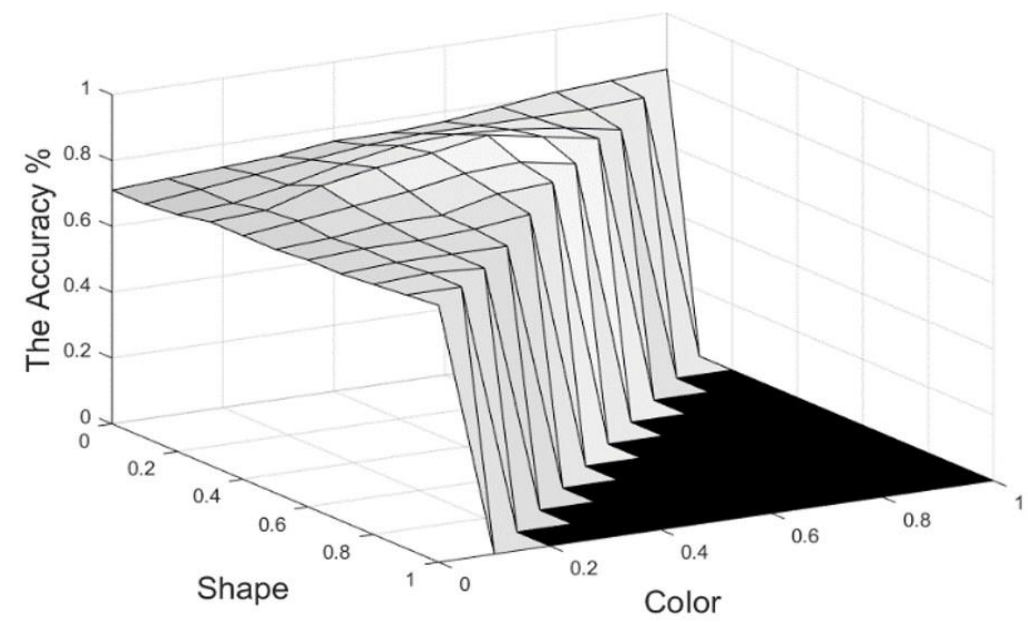

Figure 4. The varying surface of recognition accuracy rate with the feature weights

Table 1. The comparisons of precision rate between ours and the state of the art

\begin{tabular}{c|c|c|c|c|c|c}
\hline Disease name & $\begin{array}{c}\text { SVM } \\
\text { Rumpf et al., } \\
\text { 2010) }\end{array}$ & $\begin{array}{c}\text { ANN } \\
\text { (Bashish et } \\
\text { al., 2011) }\end{array}$ & $\begin{array}{c}\text { TF } \\
\text { (Arivazhagan } \\
\text { et al., 2013) }\end{array}$ & $\begin{array}{c}\text { KNN } \\
\text { (Zhang et } \\
\text { al., 2015) }\end{array}$ & $\begin{array}{c}\text { SR } \\
\text { (Zhang and } \\
\text { Wu, 2017) }\end{array}$ & Ours \\
\hline Downy mildew & 81.30 & 82.17 & 81.18 & 82.56 & 88.48 & 89.53 \\
Bacterial angular & 70.38 & 71.38 & 79.34 & 79.36 & 82.40 & 83.04 \\
Corynespora cassiicola & 71.86 & 72.20 & 76.49 & 77.68 & 84.18 & 86.41 \\
Scab & 73.19 & 70.88 & 73.65 & 73.10 & 83.15 & 84.37 \\
Gray mold & 85.35 & 87.35 & 85.20 & 86.41 & 91.29 & 93.53 \\
Anthracnose & 78.25 & 74.06 & 89.24 & 81.78 & 84.38 & 90.86 \\
Powdery mildew & 77.41 & 77.66 & 74.59 & 83.25 & 86.28 & 88.58 \\
Average & 76.82 & 76.53 & 79.96 & 80.59 & 85.74 & 88.05 \\
\hline
\end{tabular}

Table 2. The comparison of average consuming time between the state of the art algorithms and ours

\begin{tabular}{c|c|c|c|c|c|c}
\hline & SVM & ANN & TF & KNN & SR & Ours \\
\hline Average consuming time & 22.6 & 10.8 & 12.5 & 36.7 & 11.8 & 7.3 \\
\hline
\end{tabular}

\section{Discussion}

From the above experiments, we can find that in the cucumber diseases identification, our algorithm shows significant advantage in the correct recognition rate and the consuming time comparison. Next, we analyze the reasons about the above conclusion. First, the artificial neural network algorithm uses the training samples as input data to adjust the weight coefficients of the neural nodes, and uses these coefficients to fit the test sample to achieve classification. Such algorithms have higher self-adaptive and generalization ability. However, these algorithms are strongly dependent on training samples and sensitive to initial weight coefficients of neural node. In the process of calculation, they are easy to converge the local minima, thus causing misclassification. 
The SVM algorithm is a classic two-classification algorithm. It has a good generalization ability for the two-class problem in small sample environments. However, the large-scale multi-classification problem is not ideal, and the SVM algorithm needs to be solved by means of quadratic programming. The support vector will involve the calculation of the $N$-order matrix ( $N$ is the number of samples). When $N$ is large, it will take up more memory and consume a longer time. Similarly, the KNN algorithm is simple in principle and suitable for nonlinear multi-classification problems. Such algorithms are heavily dependent on data and sensitive to noise. In highdimensional data, the distance and similarity between two data points are not always. It is directly proportional, and these will affect its accuracy. At the same time, such algorithms need to calculate the distance between the test sample and each training sample, and it takes a lot of time to calculate. The SR algorithm uses the features of the sample set to sparsely represent the test features, and uses the class with the lowest reconstruction error as the classification result. This method is more accurate than other methods, but in the process of solving the sparse coefficient, the feature vector in the approximate random sample is used to fit the sparse coefficient, so that the judgment result is unstable and the recognition efficiency has room for further improvement.

All of the above algorithms use three different modality features of color, shape and texture, and all three different forms of features are simply stacked and calculated as a unified feature vector without considering these three different form features. It is against this problem that this paper designs a multi-task joint sparse representation algorithm, which requires different forms of features under the same sample set, and the sparse coefficients should have similar structures. Figure 5-A shows the sparse coefficients of the randomly extracted samples in the multi-task connection sparse representation, and Figure 5-B shows the reconstruction errors obtained by the sparse coefficients for the different disease features. In addition, the time complexity of the accelerated near-end gradient method used is $\mathrm{O}(N)$, where $N$ is the number of samples, which means that the algorithm can complete the calculation quickly.

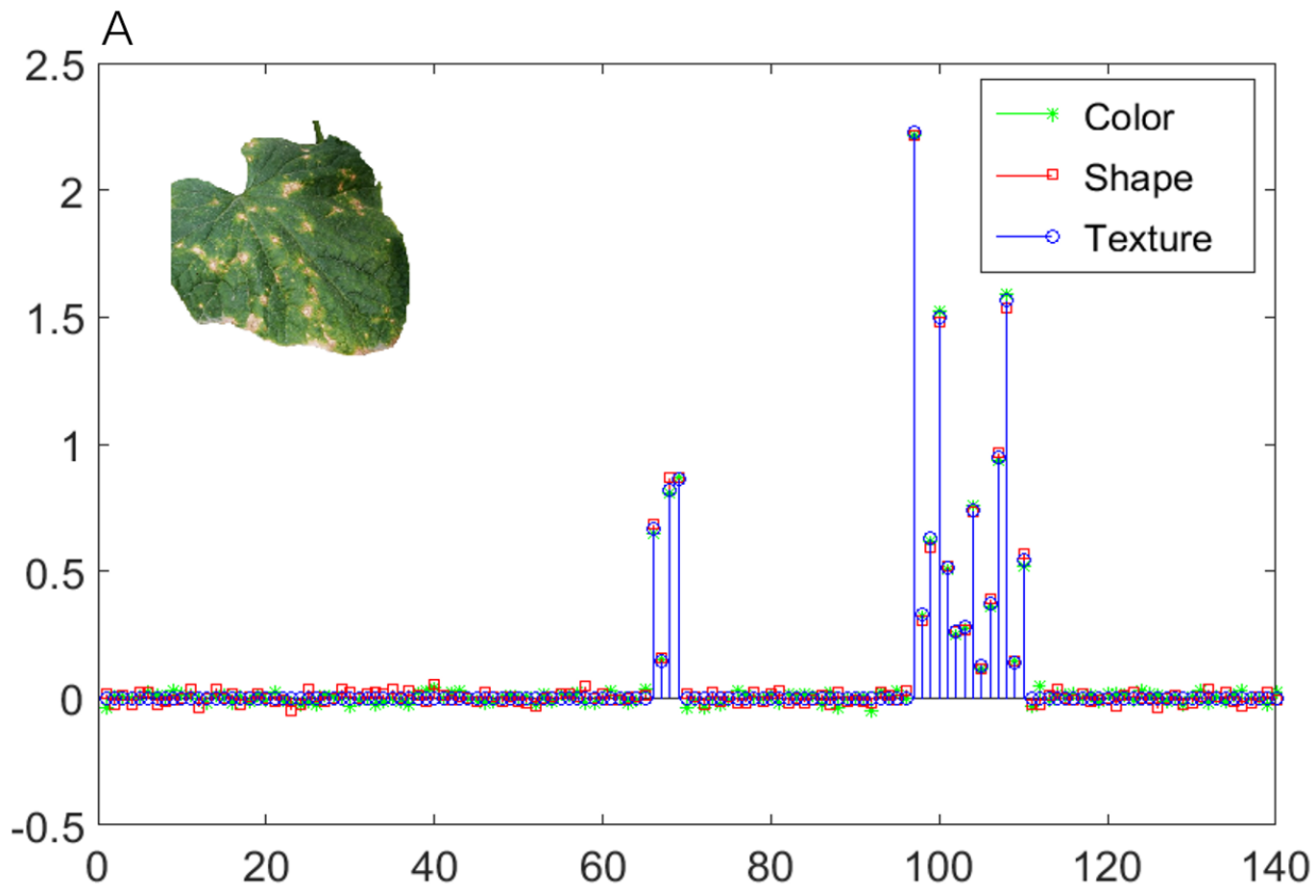




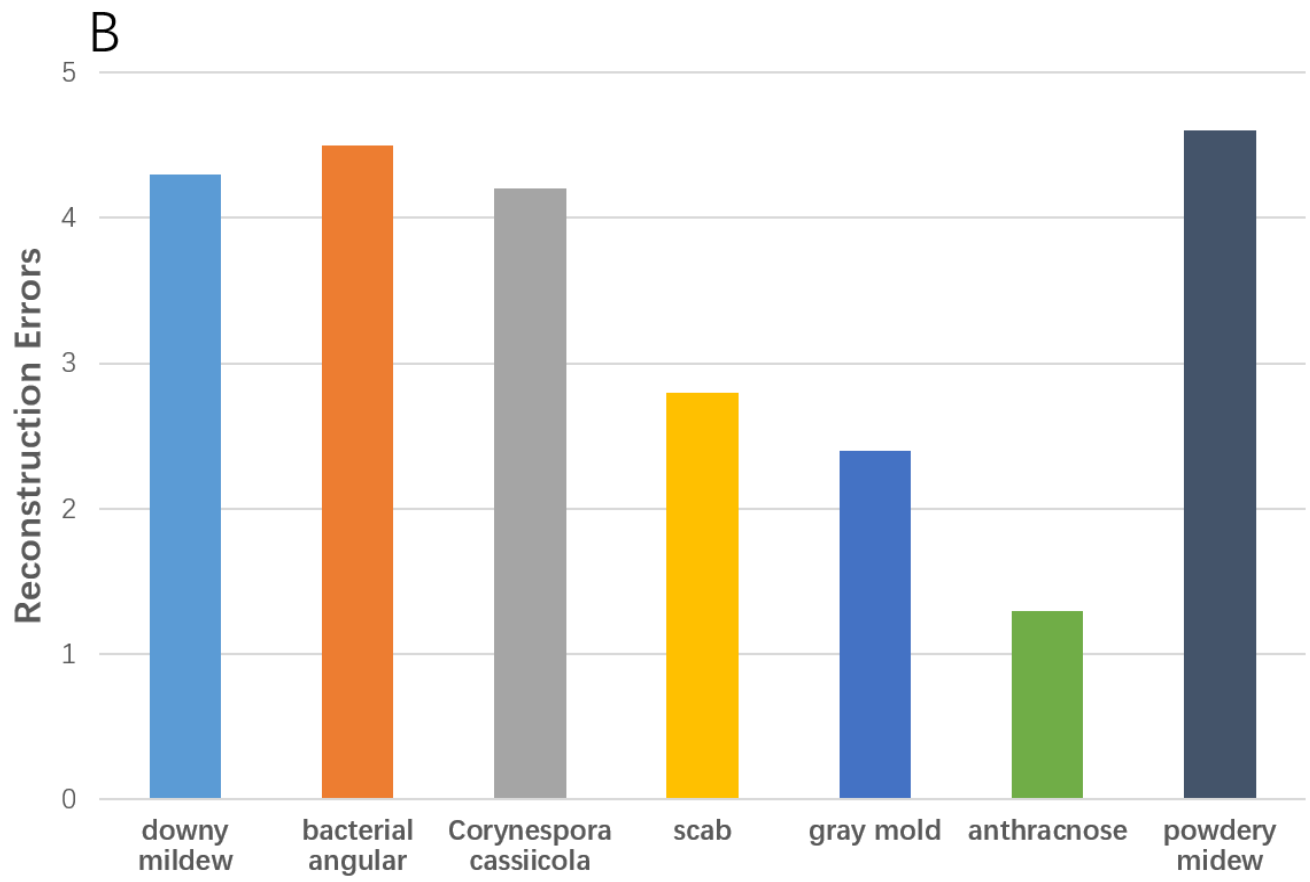

Figure 5. Multi-task joint sparse coefficient and the reconstruction error visualization of anthracnose example. A: Multi-task sparse coefficients display B: the reconstructed errors of input feature generated from different tasks with sparse coefficients

As shown in Figure 5A, the sparse features of color, shape and texture of multi-task sparse representation are approximately zero in most diseases and significantly non-zero in a small number of positions, and they have similar structures. The minimum value in $\mathrm{B}$ represents the minimum reconstruction error, that is, the classification of diseases of input samples.

\section{Conclusion}

Inspired by the idea of multi-task learning, we extracted many different types of disease features from multiple disease leaf samples for disease identification, and introduced multi-task joint sparse representation to model the data, with the commonality of different forms of disease features. Improve recognition efficiency and enhance the robustness of recognition. This algorithm is applied to the identification of cucumber diseases. Experiments show that this algorithm is superior to the disease identification algorithm based on sparse representation and other frontier algorithms that are involved in comparison in recognition rate and robustness. The recognition accuracy reaches $88.05 \%$. It can be found from the experimental part of this paper that although the approach proposed in this paper has greatly reduced the algorithm's dependence on samples, sample collection is still an indispensable step of the algorithm. At the same time, we found that the extraction of disease feature for the accurate identification also played a vital role. In the following research, we hope to obtain a wider range of leaf disease image through the unmanned aircraft system, and then extract some special features from these pictures or video to avoid the time-consuming and inefficient leaf collection step. 
Acknowledgements. This work is supported by the national natural science foundation of China (No. 61877013), the natural science foundation of Guangdong province (No. 2017A030310618) , the Key Platform and Research Project of Guangdong Province(2017GXJK073), the science and technology program of Guangdong province (No. 2016A020210131).

\section{REFERENCES}

[1] Argyriou, A., Evgeniou, T., Pontil, M. (2008): Convex multi-task feature learning. Mach. Learn 73(3): 243-272.

[2] Arivazhagan, S., Shebiah, R. N., Ananthi, S., Varthini, S. V. (2013): Detection of unhealthy region of plant leaves and classification of plant leaf diseases using texture features. - Agricultural Engineering International: CIGR Journal 15(1): 211-217.

[3] Bashish, A. D., Braik, M., Bani-Ahmad, S. Detection and classification of leaf diseases using K-means-based segmentation and neural-networks based classification. Information Technology Journal 10(2): 267-275.

[4] Bickel, S., Bogojeska, J., Lengauers, T., Scheffer, T. (2008): Multi-task learning for HIV therapy screening. - Mach Learn 23: 56-63.

[5] Caruana, R. (1997): Multi-task learning. - Mach Learn 28(1): 41-75.

[6] Dey, A. K., Sharma, M., Meshram, M. R. (2016): Image processing based leaf rot disease, detection of betel vine (Piper betle L.) - Procedia Computer Science 85: 748754.

[7] Dhingra, G., Kumar, V., Joshi, H. D. (2019): A novel computer vision based neutrosophic approach for leaf disease identification and classification. - Measurement 135: 782-794.

[8] Ghosh, S., Dubey, S. K. (2013): Comparative analysis of k-means and fuzzy c-means algorithms. - International Journal of Advanced Computer Science and Applications 4(4): 35-39.

[9] Golhani, K., Balasundram, S. K., Vadamalai, G., Pradhan, B. (2018): A review of neural networks in plant disease detection using hyperspectral data. - Information Processing in Agriculture 5: 354-371.

[10] Grinblat, G. L., Uzal, L. C., Larese, M. G., Granitto, P. M. (2016): Deep learning for plant identification using vein morphological patterns. - Computers and Electronics in Agriculture 127: 418-424.

[11] Iqbal, Z., Khan, M. A., Sharif, M. (2018): An automated detection and classification of citrus plant diseases using image processing techniques: a review. - Computers and Electronics in Agriculture 153: 12-32.

[12] Khirade, S. D., Patil, A. B. (2015): Plant disease detection using image processing. International Conference on Computing Communication Control \& Automation, IEEE Computer Society 153: 26-27.

[13] Kleynen, O., Leemans, V., Destain, M. F. (2005): Development of a multi-spectral vision system for the detection of defects on apples. - Journal of Food Engineering 69(1): 41-49.

[14] Lu, Y., Yi, S. J., Zeng, N. Y., Liu, Y. R., Zhang, Y. (2017): Identification of rice diseases using deep convolutional neural networks. - Neurocomputing 267: 378-384.

[15] Ma, J. C., Du, K. M., Zheng, F. X., Zhang, L. X., Gong, Z. H., Sun, Z. F. (2018): A recognition method for cucumber diseases using leaf symptom images based on deep convolutional neural network. - Computers and Electronics in Agriculture 154: 18-24.

[16] Mei, X., Ling, H. (2011): Robust visual tracking and vehicle classification via sparse representation. - IEEE Transactions on Pattern Analysis and Machine Intelligence 33(11): 2259-2272.

[17] Muthukannan, K., Latha, P. (2015): A PSO model for disease pattern detection on leaf surfaces. - Image Analysis \& Stereology 34(3): 209-216. 
[18] Mutka, A. M., Bart, R. S. (2015): Image-based phenotyping of plant disease symptoms. Frontiers in Plant Science 5: 734.

[19] Ozawa, S., Roy, A., Roussinov, D. (2009): A multitask learning model for online pattern recognition. - IEEE Trans Neural Netw 20(3): 430-445.

[20] Prajapati, B. S., Dabhi, V. K., Prajapati, H. B. (2016): A survey on detection and classification of cotton leaf diseases. - Electrical, Electronics, and Optimization Techniques (ICEEOT), International Conference, IEEE 2016: 2499-2506.

[21] Rao, R. P. N., Olshausen, B. A., Lewicki, M. S. (2002): Probabilistic models of the brain: Perception and neural function. MIT press. - Massachusetts Institute of Technology 220: 334.

[22] Rumpf, T., Mahlein, A. K., Steiner, U., Oerke, E. C., Dehne, H. W., Plümer, L. (2010): Early detection and classification of plant diseases with support vector machines based on hyperspectral reflectance. - Computers and Electronics in Agriculture 74(1): 91-99.

[23] Shaik, D. A., Akshay, G. G., Prashant, A. C., Parmeshwar, L. K. (2016): Intelligent autonomous farming robot with plant disease detection using image processing. International Journal of Advanced Research in Computer and Communication Engineering 5(4): 1012-1016.

[24] Sladojevic, S., Arsenovic, M., Anderla, A., Culibrk, D., Stefanovic, D. (2016): Deep neural networks based recognition of plant diseases by leaf image classification. Computational Intelligence and Neuroscience 2016: 3289801.

[25] Sun, G. L., Jia, X. L., Geng, T. Y. (2018): Plant diseases recognition based on image processing technology. - Journal of Electrical and Computer Engineering 2018: 1-7.

[26] Unay D, Gosselin B, Kleynen O, Leemans, V., Destainc, M., Debeir, O. (2011): Automatic grading of Bi-colored apples by multispectral machine vision. - Computers and Electronics in Agriculture 75(1): 204-212.

[27] Wright, J., Yang, A. Y., Ganesh, A., Sastry, S. S., Ma, Y. (2009): Robust face recognition via sparse representation. - IEEE Transactions on Pattern Analysis and Machine Intelligence 31(2): 210-227.

[28] Yang, J. C., Wright, J., Huang, T. S., Ma, Y. (2010): Image super-resolution via sparse representation. - IEEE Transactions on Image Processing 19(11): 2861-2873.

[29] Zhang, S., Wang, Z. (2016): Cucumber disease recognition based on Global-Local Singular value decomposition. - Neurocomputing 205: 341-348.

[30] Zhang, S., Huang, W., Zhang, C. (2019): Three-channel convolutional neural networks for vegetable leaf disease recognition. - Cognitive Systems Research 53: 31-41.

[31] Zhang, S. W., Shang, Y. J., Wang, L. (2015): Plant disease recognition based on plant leaf image. - Journal of Animal \& Plant Sciences 25(3): 42-45.

[32] Zhang, S. W., Wu, X. W., You, Z. H., Zhang, L. Q. (2017a): Leaf image based cucumber disease recognition using sparse representation classification. - Computers and Electronics in Agriculture 134: 135-141.

[33] Zhang, S. W., Zhu, Y. H., You, Z. H., Wu, X. W. (2017b): Fusion of super-pixel, expectation maximization and PHOG for recognizing cucumber diseases. - Computers and Electronics in Agriculture 140: 338-347.

[34] Zhang, S. W., Wang, H. X., Huang, W. Z., You, Z. H. (2018): Plant diseased leaf segmentation and recognition by fusion of superpixel, K-means and PHOG. - OptikInternational Journal for Light and Electron Optics 157: 866-872. 


\section{APPENDIX}

Feature list

\begin{tabular}{|c|c|c|c|}
\hline Type & Index & Name & Formula \\
\hline \multirow{4}{*}{ Color } & $C_{l}$ & Mean & $\mu_{L}=\sum b p(b)$ \\
\hline & $C_{2}$ & Variance & $\sigma_{L}^{2}=\sum\left(b-C_{1}\right)^{2} p(b)$ \\
\hline & $C_{3}$ & Energy & $E_{L}=\sum(p(b))^{2}$ \\
\hline & $C_{4}$ & Standard Color Histogram & $h_{C_{I}}=p\left(p \in I_{C_{I}}\right)=\frac{H_{C_{l}}(I)}{A}$ \\
\hline \multicolumn{4}{|c|}{$p(b)$ : the probability of the level $b$} \\
\hline \multirow{7}{*}{ Shape } & $S_{1}$ & Rectangularity & $S_{1}=A / S_{M B R}$ \\
\hline & $S_{2}$ & Compactness & $S_{2}=4 \pi A / P^{2}$ \\
\hline & $S_{3}$ & Elongation & $S_{3}=\frac{M R B^{\prime} S \text { width }}{M R B^{\prime} S \text { length }}$ \\
\hline & $S_{4}$ & Circularity & $S_{4}=\left(4 A M R B^{\prime} S\right.$ length $) / \pi$ \\
\hline & $S_{5}$ & Sphericity & $S_{5}=$ Inscribed Radius \\
\hline & & 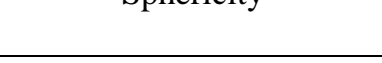 & $\mathrm{S}_{5}-\overline{\text { Circumscibed Radius }}$ \\
\hline & $S_{6}$ & Lobation & $S_{6}=R_{1} / M$ \\
\hline
\end{tabular}

A: the area of diseased region; $S_{M B R}$ : the areas of minimum bounding rectangles; P: Lesion length; MRB: minimum bounding rectangles; $\mathrm{M}$ : The shortest distance from the center of mass of a region to the

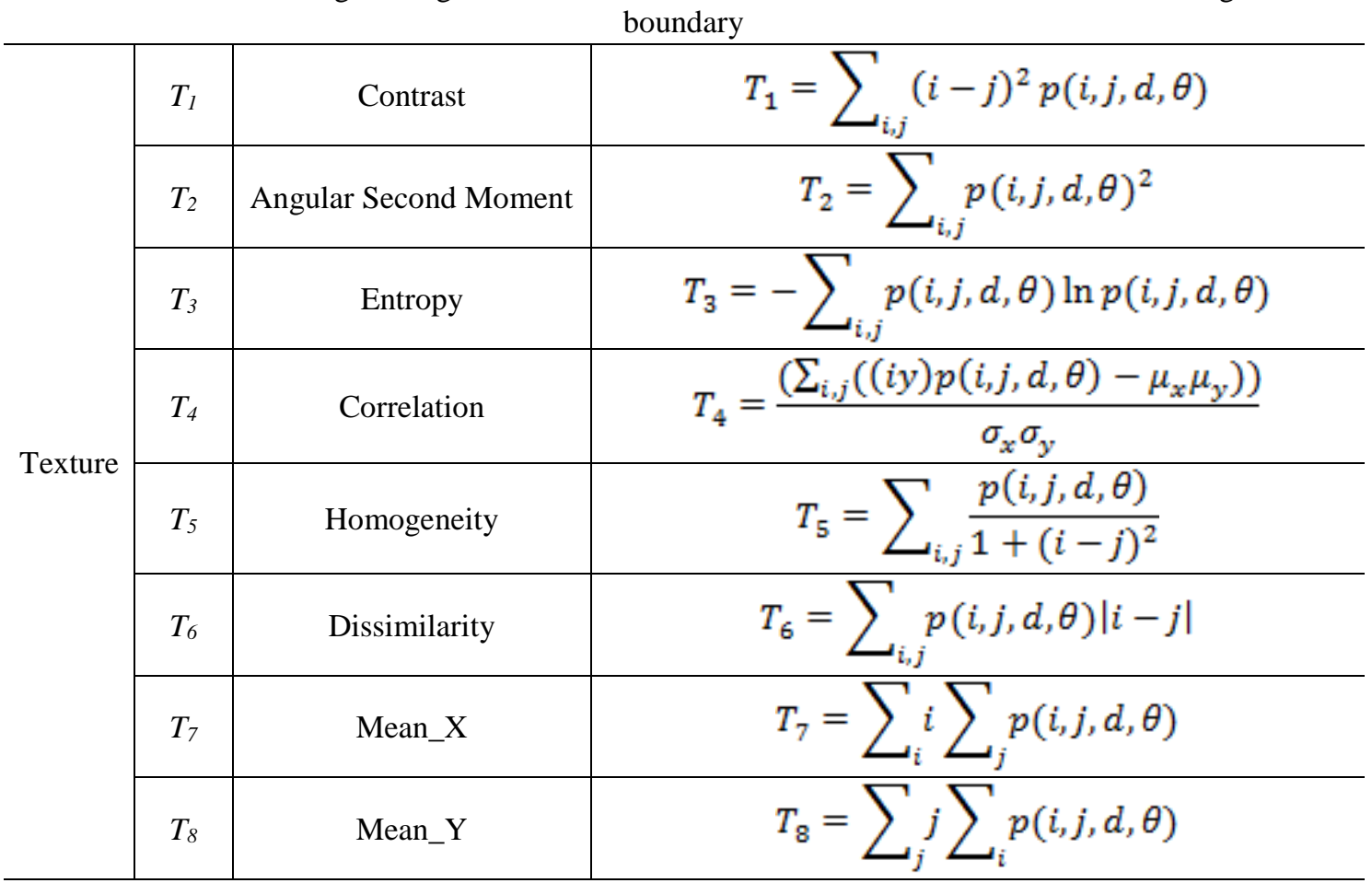




\begin{tabular}{|c|c|c}
\hline$T_{9}$ & Variance_X & $T_{9}=\sum_{i}\left(j-T_{7}\right)^{2} \sum_{j} p(i, j, d, \theta)$ \\
\hline$T_{10}$ & Variance_Y & $T_{10}=\sum_{i}\left(j-T_{8}\right)^{2} \sum_{j} p(i, j, d, \theta)$ \\
\hline$T_{12}$ & Inverse Difference & $T_{12}=\sum_{i, j} \frac{p(i, j, d, \theta)}{1+|i-j|}$ \\
\hline$T_{13}$ & $\begin{array}{c}\text { Inverse Different Moment } \\
\text { Normalized }\end{array}$ & $T_{13}=\sum_{i, j} \frac{p(i, j, d, \theta) N^{2}}{N^{2}+(i-j)^{2}}$ \\
\hline$T_{14}$ & Sum Average & $T_{13}=\sum_{i=0}^{2(N-1)} i P_{x+y}(i)$ \\
\hline$T_{15}$ & Sum Entropy & $T_{15}=-\sum_{i=0}^{2(N-1)} i P_{x+y}(i) \log \left(P_{x+y}(t)\right)$ \\
\hline$T_{16}$ & Sum Variance & $T_{16}=-\sum_{i=0}^{2(N-1)}(i-S E N T)^{2} P_{x+y}(i)$ \\
\hline$T_{17}$ & Difference Entropy & $T_{17}=\sum_{i=0}^{N-1} P_{x-y}(t) \log \left(P_{x-y}(i)\right)$ \\
\hline$T_{18}$ & Maximum Probability & $T_{18}=\sum_{i, j}^{\max (p(i, j, d, \theta))}$ \\
\hline
\end{tabular}

